معرفة تأثير إحلال مستويات مختلفة من مجروش نوى التمر مع أو بدون إضافة خميرة الخبز Saccharomyces cerevisiae

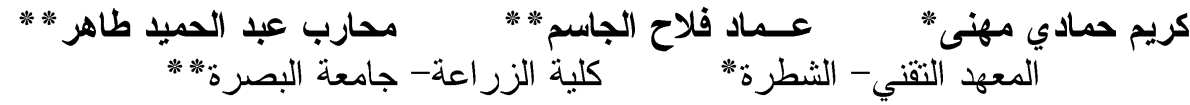

الخلاصة

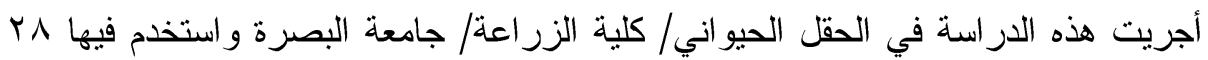

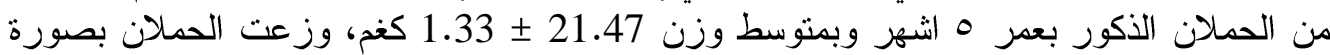
عشو ائية ومتساوية على سبعة مجاميع تغذوية لار اسة نمو وتسمين الحملان العر ابية ولمدة ، 9 يوم وكانت العلائق التجريبية كالاتي:العليقة الاولى (السيطرة): غذيت الحملان عليقة مركزة فقط وبنسبة بانة من من وزن الجسم.

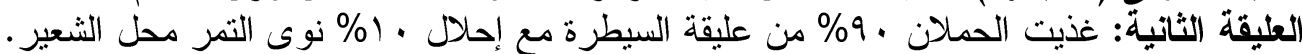

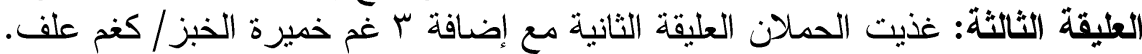

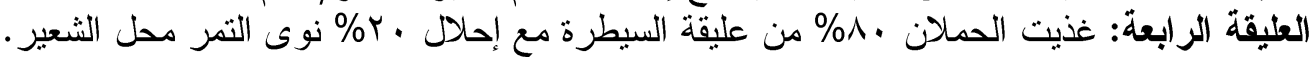

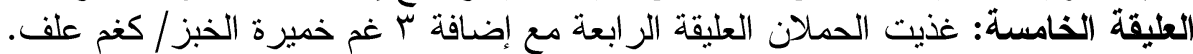

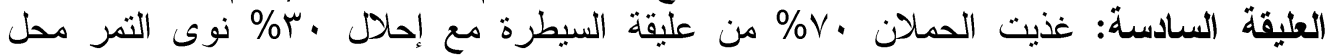

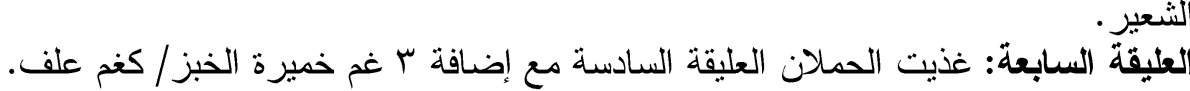
توصلت الار اسة إلى النتائج الأثية:-

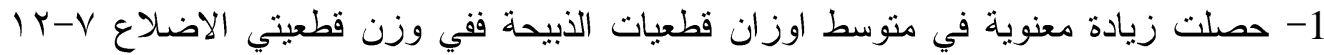

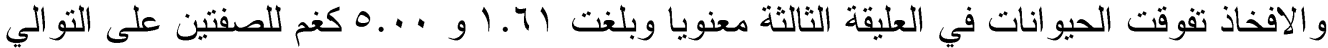

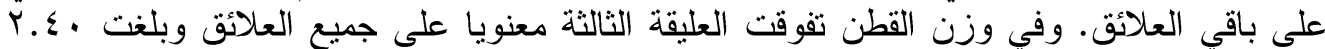

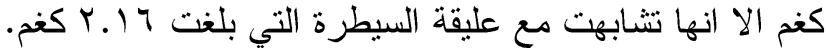
2- وجدت فروق معنوية في بعض قياسات الذبيحة ففي محيط الفخذ تفوقت العليقتان الثانية و الثالثة

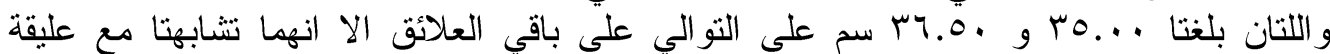

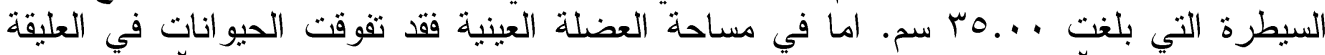

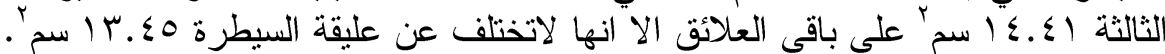

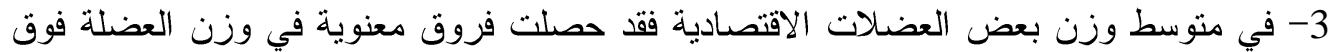

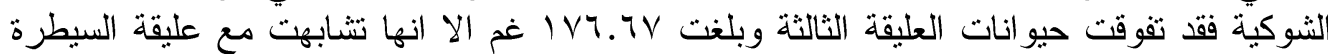

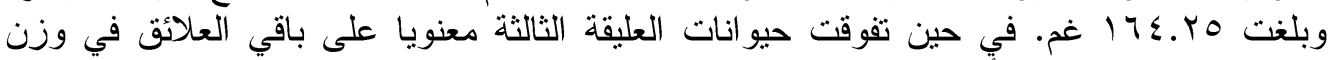

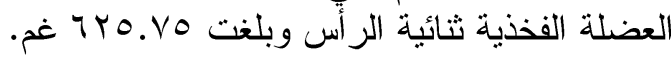

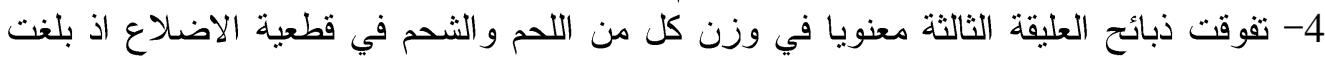

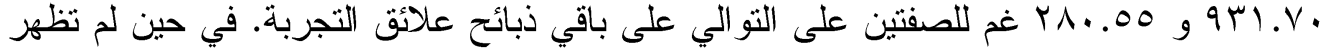

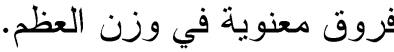
5- في متوسط وزن الدهون المفصولة الكلية تفوقت العليقتان الثانية والثالثة معنويا واللتان بلغتان الثانيا r.9 أو 1.90 1.90

الكلمات المفتاحية: خميرة الخبز، نوى التمر، صفات الذبيحة، الحملان العر ابية.

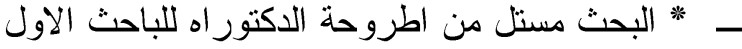

Fayoum J. Agric. Res. \& Dev., Vol. 33, No.2, July, 2019 


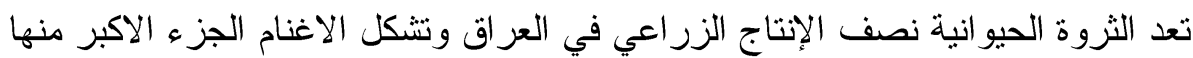

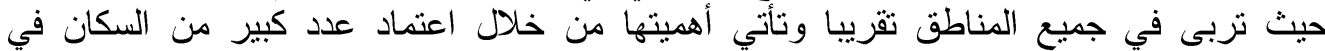

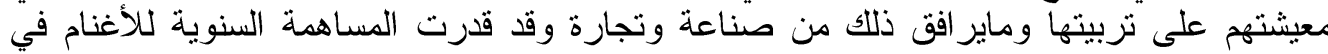

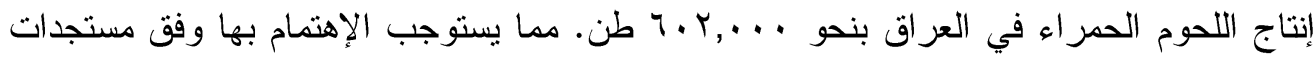

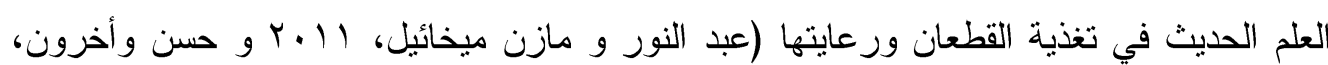
.$(r \cdot 1)$ يعتبر العراق من البلدان المنتجة للتمور وذلك لوجود أعداد كبيره من النخيل المزروعة في أراضيه وقد قدرت بأكثر من 12,396,000 نخلة تنتج سنويا بساء ألف طن من التمور (وزارة

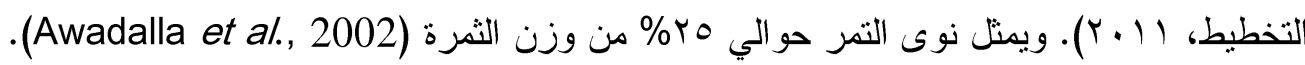

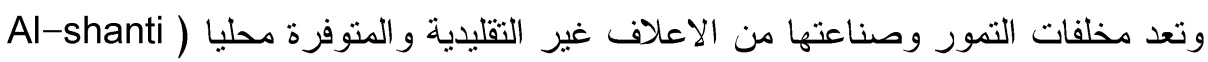

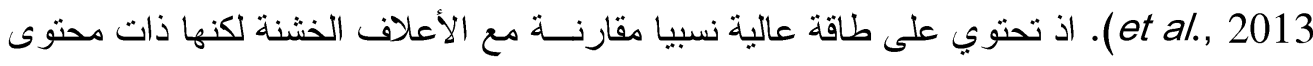

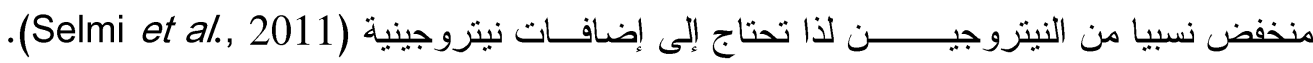

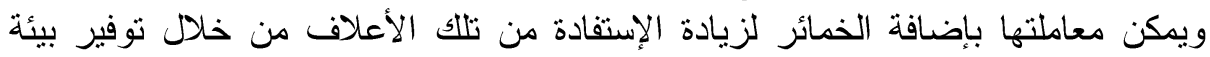

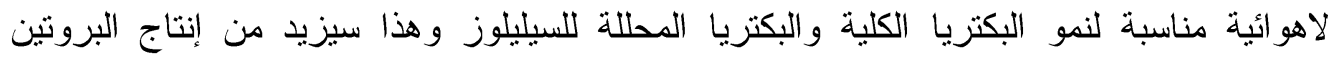

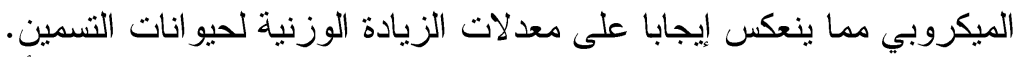

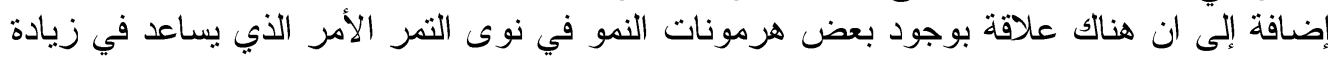

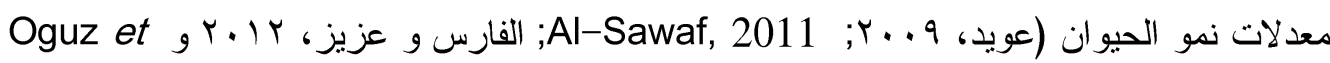
.(al., 2015

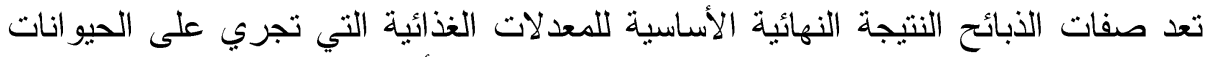

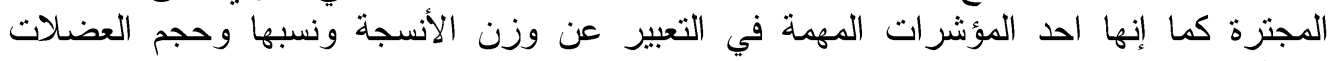
و القطعيات المختلفة للذبائح وتعد الذبيحة عاملاّ مهما في تقويم كفاءة الإنتاج الكمي و النوني وني في اللحم

.(Payandeh and Khafilzadh, 2007) وقد هدفت هذه الدراسة إلى معرفة تاثير إحلال مستويات مختلفة من مجروش نوى التمر مع أو

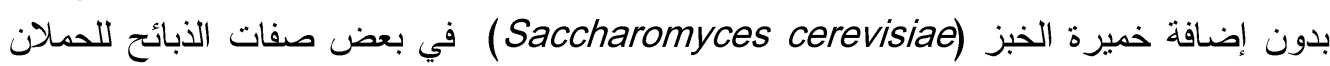

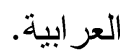

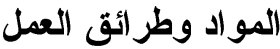

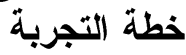
أجريت هذه الدراسة في الحقل الحيو اني التابع إلى محطة الأبحاث الزر اعية/ كلية الزر اعة/ جامعة

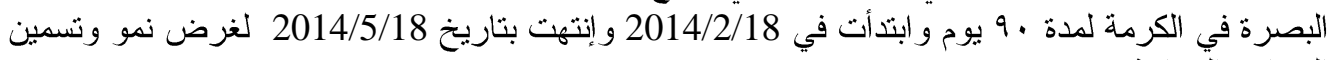

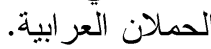

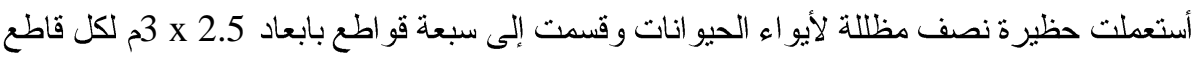
تجربة النمو والتسمين

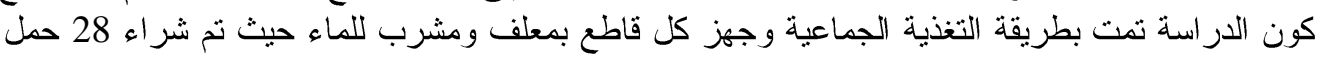

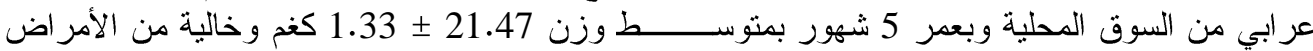

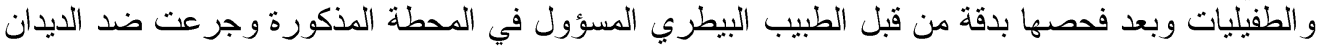
الداخلية والخارجية كما لقحت ضد مرض فئل التسمم المعوي وقسمت بشكل عشو ائي إلى سبعة مجاميع تغذوية الطية

Fayoum J. Agric. Res. \& Dev., Vol. 33, No.2, July, 2019 
rr

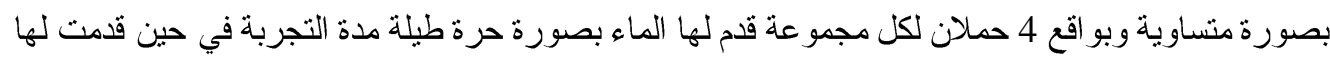

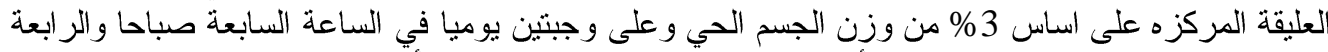

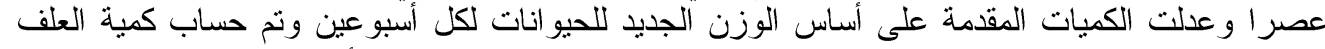

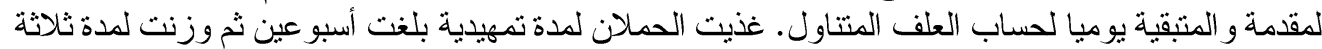

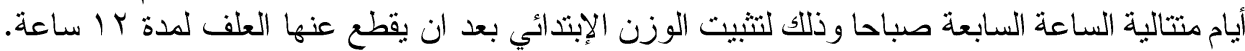

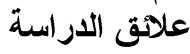

تم تكوين سبعة علائق تحتوي على نسب مختلفة من الشعير ونخالة الحنطة ونوى التمر و اليوريا

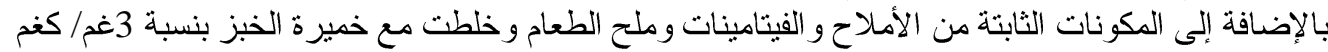
مادة علفية وتوضح الجداول ( او ب r) التركيب الكيميائي ونسب المو اد العلفية ومكوناتها للعلائق المستخدمة في

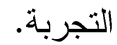

جدول. (1): مكونات العلاثق المركزة من المواد الاولية (\%).

\begin{tabular}{|c|c|c|c|c|c|c|c|}
\hline \multicolumn{7}{|c|}{ نسبة الماده العلفية الداخله في العلائق التجريبية \% } & \multirow[b]{2}{*}{ المواد الأولية } \\
\hline 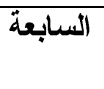 & السادسة & الخامسة & الز ابعة & الثالثة & الثانية & (النيطرة) & \\
\hline$r$. & $r$. & 40 & 40 & 50 & 50 & 60 & شعير \\
\hline 35 & 35 & 35 & 35 & 35 & 35 & 35 & ن نخالة حنطة \\
\hline 1 & 1 & 1 & 1 & 1 & 1 & 1 & يوريا \\
\hline 30 & 30 & 20 & 20 & 10 & 10 & - & نوى تمر \\
\hline 3 & 3 & 3 & 3 & 3 & 3 & 3 & فيتامينات ومعادن " \\
\hline 1 & 1 & 1 & 1 & 1 & 1 & 1 & ملح طعام \\
\hline 3 & - & 3 & - & 3 & - & - & خميرة خبز 3غم/ كغم علف \\
\hline
\end{tabular}

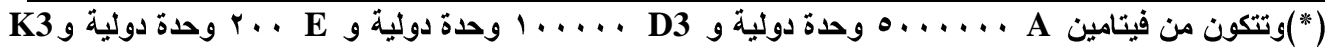

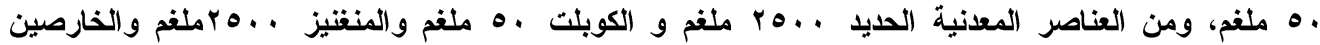

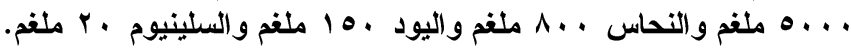

جدول. (r): التركيب الكيميائى لعلاثق التجربة على اساس المادة الجافة.

\begin{tabular}{|c|c|c|c|c|c|c|c|}
\hline \multicolumn{7}{|c|}{ العلائق } & \multirow[t]{2}{*}{ التركيب الكيميائي (\%) } \\
\hline السابعة & السادسة & الخامسة & الر ابعة & الثالثة & الثاتية & الاولى & \\
\hline 93.48 & 93.48 & 93.32 & 93.32 & 93.17 & 93.17 & 94.32 & المادة الجافة \\
\hline 12.48 & 12.48 & 12.51 & 12.51 & 12.98 & 12.98 & 12.90 & البروتين الخام \\
\hline 2.40 & 2.40 & 2.12 & 2.12 & 1.75 & 1.75 & 1.13 & مستخلص الايثر \\
\hline 10.63 & 10.63 & 9.71 & 9.71 & 8.53 & 8.53 & 7.50 & الالياف الخام \\
\hline 64.40 & 64.40 & 65.64 & 65.64 & 65.68 & 65.68 & 66.38 & المستخلص الخالي من \\
\hline 89.91 & 89.91 & 89.98 & 89.98 & 88.94 & 88.94 & 87.91 & المادة|العضوية \\
\hline 3.57 & 3.57 & 4.04 & 4.04 & 4.23 & 4.23 & 4.41 & الرماد \\
\hline 9.099 & 9.099 & 9.125 & 9.125 & 9.262 & 9.262 & 9.351 & الطاقة المتأيضة ميكاجول/ كغم \\
\hline
\end{tabular}

- حسبت الطاقة المتأيضة للعلاثق التجريبية السبعة وفقا لمعادلة وزارة الزراعة الاسكتلندية ( , MAFF

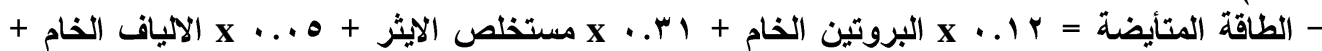

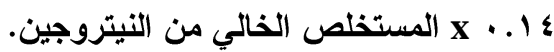

Fayoum J. Agric. Res. \& Dev., Vol. 33, No.2, July, 2019 
عمليات الأبح ودر اسة صفات الأبائح

بعد انتهاء مدة النمو و التسمين ذبحت الحيو النات التحة بعد أن صومت لمدة 18 ساعة فيما ترك الماء

متوفر اللحملان إلى حين إجراء عملية الذبح. بعد ذبح الحيوانات وسلخها ونات وتفريغ محتويات القناة

الوضنمية سجلت القياسات التالية:

أوزان قطعيات الأبيحة

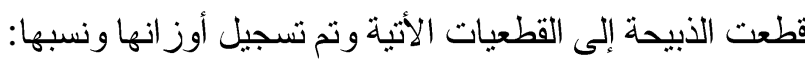

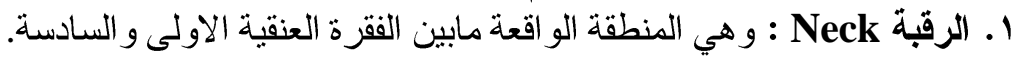

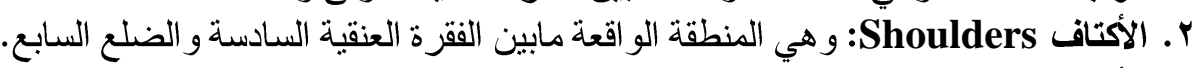

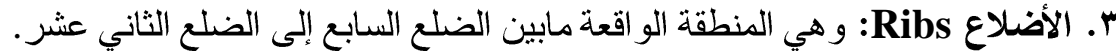

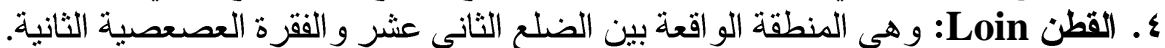

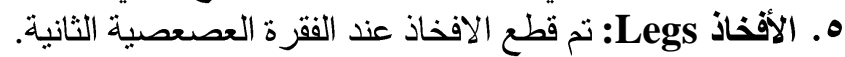

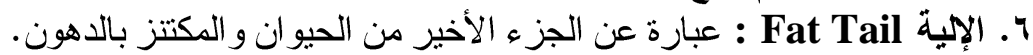
قياسات الأبيحة وتثمل طول الأبيحة وعمق الصدر ومحيط الفخذ ومساحة العضلة العينية وسمك الطبقة الاهنية تم قياس طول الذبيحة من الطرف الامامي للضلع الأول إلى الطرف الامامي لعظم

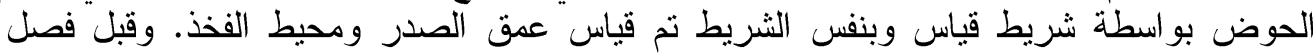

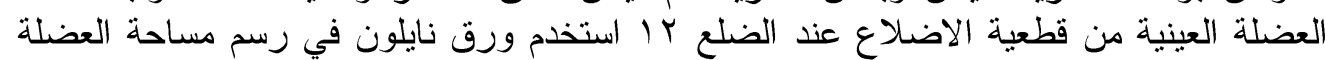

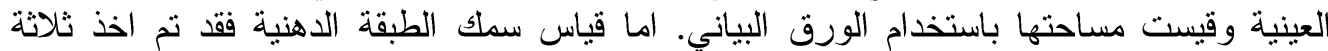

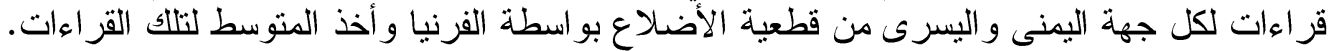

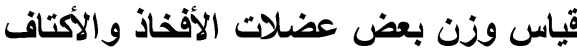
أزيلت العضلة الفخذية ثنائية الر أس (Biceps femoris) من كل فخذ والأني ونم وزنها وكذلك أزيلت

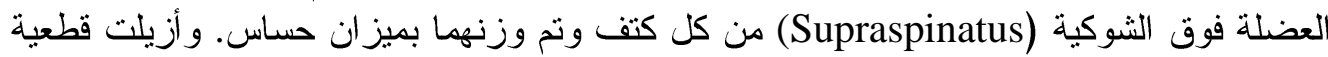
الأضلاع وجمدت لحين الإستعمال. الفصل الفيزيائي لقطعية الاضلاع الإعلاع

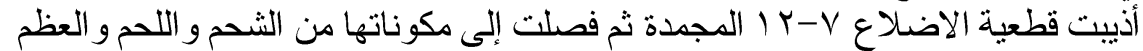
وذلك بأستخدام مشارط طبية وسكاكين كما حسبت أوزان كل من هذه الأن المكونات كما فصلت العضلة

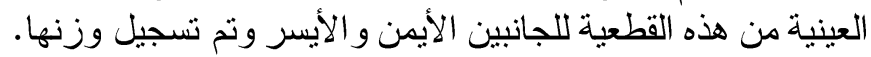

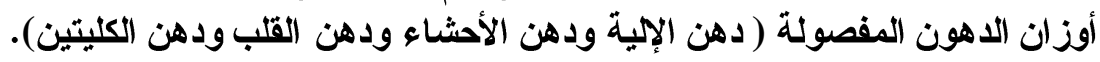
التحليل الإحصائي التهان

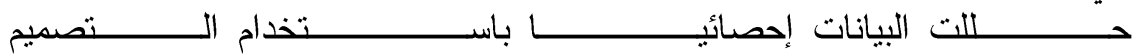
العشو ائي الكامــــل (Completely Randomized Design : CRD) لسبعة معاملات و اختبرت

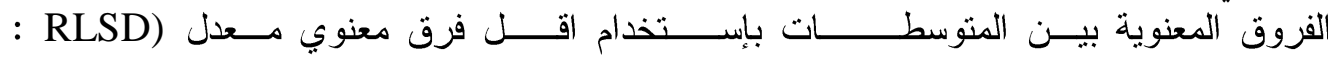
(SPSS , باستخدام البرنامج الاحصائي الجاهز (Revised Least Significant Differences

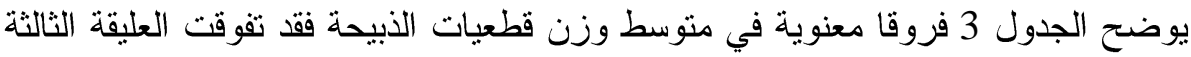

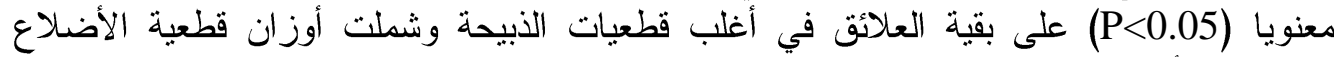

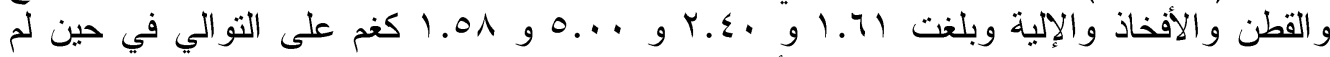

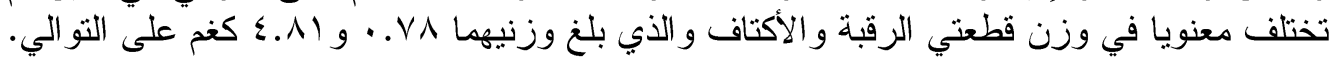

Fayoum J. Agric. Res. \& Dev., Vol. 33, No.2, July, 2019 
r $\varepsilon$

كما يوضح جدول 10 بأن العلائق الخامسة والسادسة والسابعة (.r و •r) \% نوى التمر جاءت بالمرتبة الأخيرة وقد يعزى سبب وجود الفروق المعنوية في قطعيات الذبيحة لوجود علاقة طرونة طودية بين أوزان الذبيحة و أوزان القطعيات ونسب التصافي التي تحسنت عند إستخدام الخميرة مع نسبة ، ا\% من نوى التمر حيث تعمل الخميرة على زيادة كفاءة تمثيل العناصر الغذائية وزيادة المتتاول من المادة العلفية وزيادة جاهزية إمتصاص العناصر المعدنية وزيادة ذوبانها و إمتصاصها

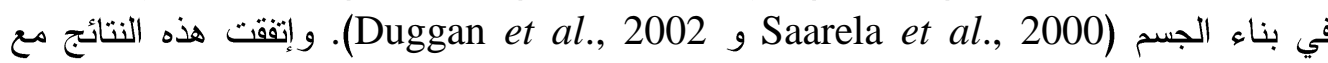
.Abdul-Aziz et al. (2001) جدول(ץ). متوسط أوزان قطعيات الأبيحة للعلاثق التجريبية المختلفة 土 الخطأ القياسي.

\begin{tabular}{|c|c|c|c|c|c|c|}
\hline \multicolumn{6}{|c|}{ الصفات 土 الخطأ القياسي } & \multirow[t]{2}{*}{ العلائق } \\
\hline وزن الإلية (كغم) & وزن الأفخاذ (كغم) & وزن القطن & وزن قطعية الأضلاع & وزن الأكتاف & وزن الرقبة & \\
\hline$a \cdot . \leqslant 0 \pm 1 . v \varepsilon$ & 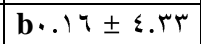 & 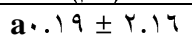 & b...9 $\pm 1 . \varepsilon \varepsilon$ & $.0 Y \pm \varepsilon .0\}$ & $\cdots \varepsilon \pm \cdot . \wedge$ & الأولى (السيطرة) \\
\hline$a \cdot . \leqslant r \pm 1 . \leqslant 9$ & b..11 $\pm \ldots .9$ & b..Ir $\pm Y . . T$ & b...t $\pm 1 . \varepsilon V$ &.$\leqslant 9 \pm \varepsilon .7$. & $. .0 \pm \ldots v \varepsilon$ & 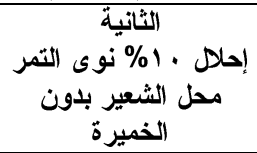 \\
\hline$a \cdot . r V \pm 1.01$ & $a+.1 r \pm 0 .$. & a..17 \pm r.s. & a... $4 \pm 1.71$ & $. . M V \pm \varepsilon . \wedge 1$ & $\ldots r \pm \ldots v \wedge$ & 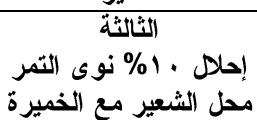 \\
\hline b..IT $\pm 1 . r V$ & b..IT $\pm \varepsilon \ldots$ & b. $.10 \pm 1.99$ & b...9 \pm 1.0. & $. . \varepsilon \Gamma \pm \varepsilon . r \Lambda$ & $. . r \pm \ldots v T$ & 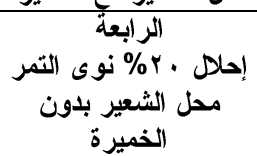 \\
\hline$a \cdot . r o \pm 1 . r \varepsilon$ & b.. $\leq \leq \pm \leq . Y$. & b..Ir \pm r.A & b...0 $\pm 1 . \leqslant \wedge$ & $. . r q \pm \varepsilon .00$ & $. . r \pm . .10$ & محل الحلا ، الخعير مـ نوى الخمر الخميرة \\
\hline b.. $\varepsilon 0 \pm 1 . r K$ & $c . . I V \pm r . \Lambda$. & c..r. $\pm 1 . \wedge 0$ & $d \cdot . . \varepsilon \pm 1 . r \wedge$ & $\cdot r \cdot \pm \varepsilon .1 \varepsilon$ & $. .0 \pm . . \wedge \mu$ & إحلا · السادسة نوى التمر \\
\hline 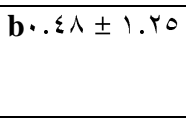 & bc..19 \pm 1.97 & c..1V $\pm 1.9 r$ & c...9 \pm 1.14 & $. \mu \wedge \pm \varepsilon . \mu$ & $. .0 \pm . .10$ & محل الحلا · الشعير مـ نوى التمر الخميرة \\
\hline \% & * & $*$ & $*$ & N.S & N.S & مستوى المعنوية \\
\hline
\end{tabular}

(") المتوسطات التي تحمل حروف مختلفة ضمن الصفة الواحدة عموديا تختلف معنويا عند مستوى (0 . . +).

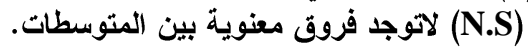

يوضح الجدول ؛ عدم وجود فروق معنوية في طول الذبيحة وعمق الصدر بين العلائق التجريبية قياسات الأبيحة

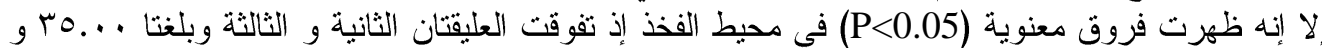

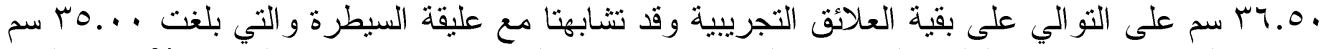

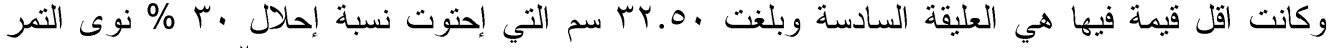

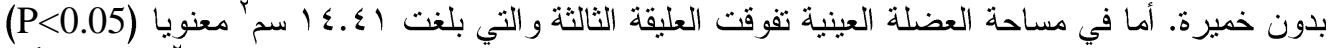

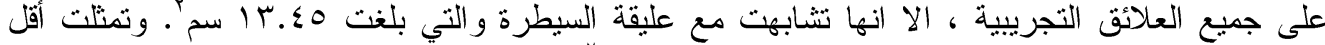

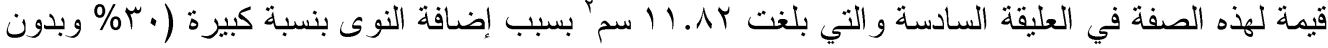

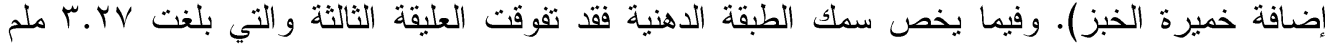

Fayoum J. Agric. Res. \& Dev., Vol. 33, No.2, July, 2019 


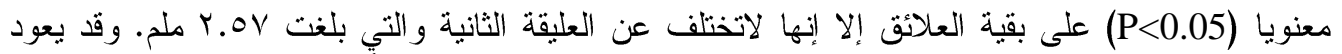

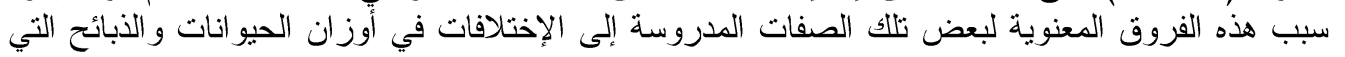

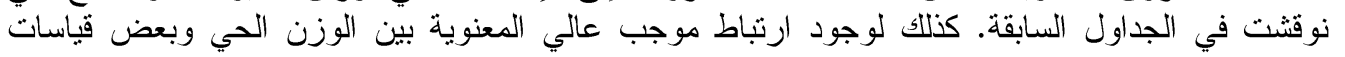

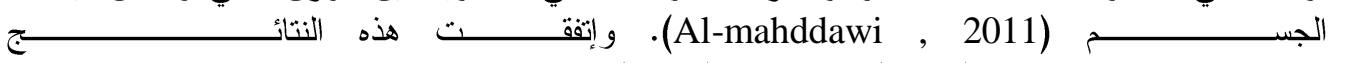

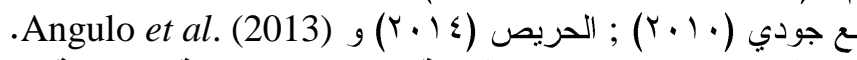

جدول (ع). متوسط قياسات بعض صفات الأبيحة للعلانق التجريبية المختلفة 土 الخطأ القياسي.

\begin{tabular}{|c|c|c|c|c|c|}
\hline \multicolumn{5}{|c|}{ الصفات 土 الخطأ القياسي } & \multirow[t]{2}{*}{ العلاثق } \\
\hline سمك الطبقة الدهنية & مساحة العضلة العينية & محيط الفخذ (سم) & عمق الصدا (سم) & طول الأبيحةٌ (سم) & \\
\hline 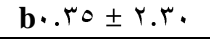 & ab $1.90 \pm 1 T . \leq 0$ & a).. \pm . $0 . .$. & $1 \ldots \pm r 1.0$. & $r .1 \cdot \pm T 1 . r_{0}$ & ألاولى (السيطرة) \\
\hline ab..rq \pm r.ov & bc $1.1 V \pm I Y . T$. & a).Tr \pm ro... & $\because 4 \pm Y . .0$. & $1 . V I \pm T Y . Y_{0}$ & 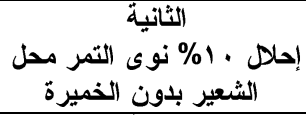 \\
\hline a..sI \pm T.YV & a).YA $\pm 1 \leq . \leq 1$ & a). $1 \leq \pm$ พч.0. & $1 . \Lambda V \pm Y Y . Y_{0}$ & $1 . \wedge r \pm Y r . .$. & 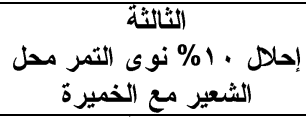 \\
\hline bc..01 \pm r... & bc $1.1 Y \pm 1 r . \leqslant 9$ & b). $99 \pm$ rs.o. & $1.0 r \pm r . . \leqslant V$ & r.01 & 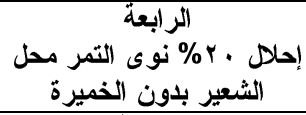 \\
\hline bc..ry $\pm 1.9 r$ & bc $1 . r T \pm I r . r \wedge$ & b)...$\pm \pm \leqslant .0$. & $1.0 . \pm r_{. . r_{0}}$ & $1 . \Sigma 1 \pm \pi T_{.}$. & 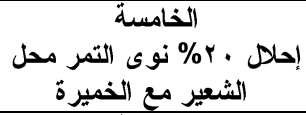 \\
\hline c..rV $\pm 1.7 r$ & cl.ro $\pm 11 . \wedge r$ & cl. $\{1 \pm$ KY.O. & $1 . \Sigma 1 \pm r \ldots$. & $r \ldots \pm \uparrow . .0$. & 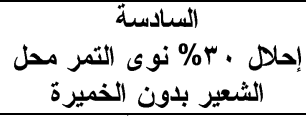 \\
\hline c..os $\pm 1.0 r$ & bc).イ. \pm IY.।^ & br... \pm rs... & $1 . V^{\prime} \pm r_{.} r_{0}$ & r. $\leqslant \leqslant \pm 09 . \wedge$. & 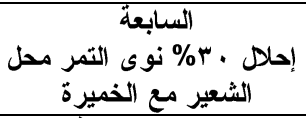 \\
\hline$*$ & * & * & N.S & N.S & مستوى المعنوية \\
\hline
\end{tabular}

\footnotetext{
(") المتوسطات التي تحمل حروف مختلفة ضمن الصفة الواحدة عموديا تختلف معنويا عند مستوى (ه ...). (N.S)
}

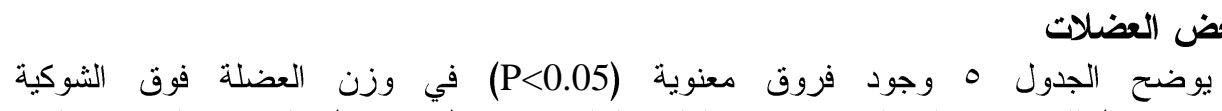

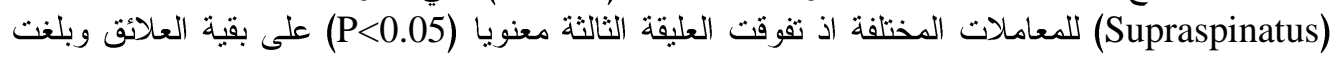
VY.TV

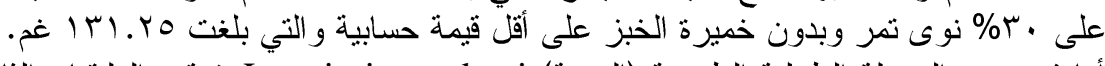

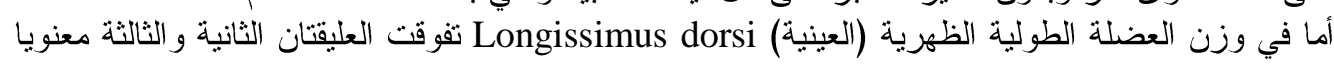
(P<0.05)

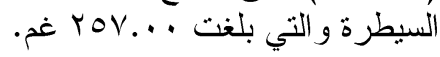

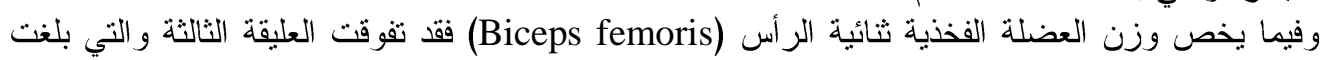
بالع Tro.Vo

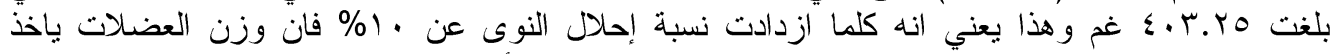

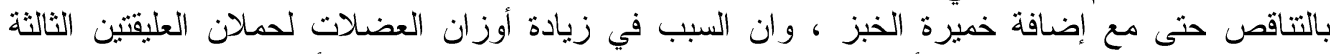

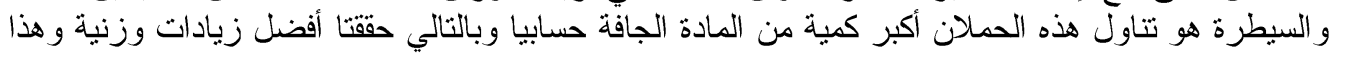

Fayoum J. Agric. Res. \& Dev., Vol. 33, No.2, July, 2019 
rч

يعود إلى كفاءة استفادتها من العليقة مما ترجمته تلك الحمالن إلى زيادة وزنية في عضلاتها المذكورة

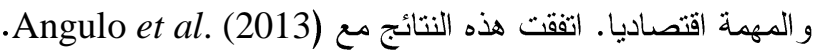

جدول (•). متوسط أوزان بعض العضلات للعلاثق التجريبية المختلفة 土 الخطأ القياسي.

\begin{tabular}{|c|c|c|c|}
\hline \multicolumn{3}{|c|}{ الصفات 土 الخطأ القياسى } & \multirow[t]{2}{*}{ العلاثق } \\
\hline وزن العضلة الفخذيةٌ الثنائية & وزن العضلة الطولية الظهرية & وزن العضلة فوق الشوكية & \\
\hline b) $\leqslant . V Y \pm \leqslant V V .0$. & ar4.人т \pm rov... & a)Y.T. \pm IYะ.ro & الاولى (السيطرة) \\
\hline 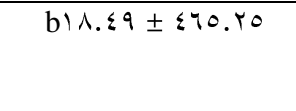 & $a r q .1 V \pm r 0 . .0$. & b)V.qr $\pm 1 r \Lambda . r_{0}$ & 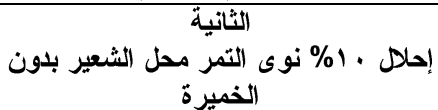 \\
\hline a)A.VY \pm TYO.VO & 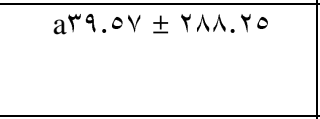 & a)V.SY \pm IVT.TV & إحلال · 1\% نوى الثمر محل الشعير ميع \\
\hline c) $1 . . . \pm \leqslant \leqslant \Lambda . \vee 0$ & brร.Аq \pm r & b)1..1 \pm 119.0. & 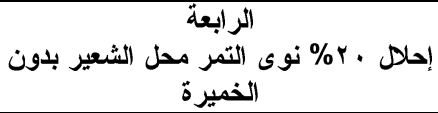 \\
\hline b)r.q1 $\pm\{4 r . r r$ & $b^{r} \cdot .11 \pm r \leqslant 1 . r_{0}$ & b) $9.19 \pm 119.10$ & إحلال · r\% نوى الثمر محل الشعير ميع \\
\hline dIT.l. \pm E.r.Yo & $\mathrm{br}^{r} . \mathrm{rq} \pm$ rYA.ro & b) $9.0 \leq \pm 1 \% 1 . r_{0}$ & إحلال ، ٪\% نوى التمر محل الشعير بدون \\
\hline 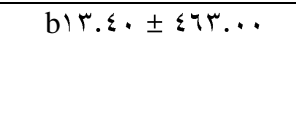 & $\mathrm{br}_{\mathrm{L} . .1 \cdot \pm \text { rro.ro }}$ & bI Y.VY \pm I $\leq \varepsilon \ldots$ & إحلال · r\% نوى التمرة محل الثعير مع \\
\hline * & * & * & مستوى المعنوية \\
\hline
\end{tabular}

(") المتوسطات الثي تحمل حروف مختلفة ضمن الصفة الواحدة عموديا تختلف معنويا عند مستوى (ه ..).)

Fayoum J. Agric. Res. \& Dev., Vol. 33, No.2, July, 2019 
الفصل الفيزيائي لقطعية الأضلاع

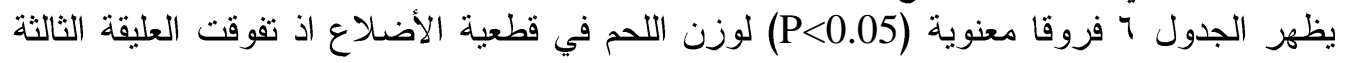

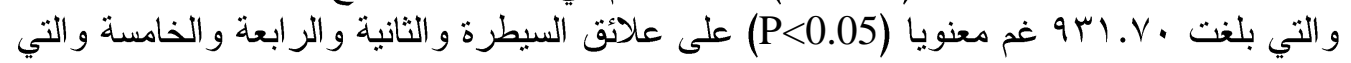

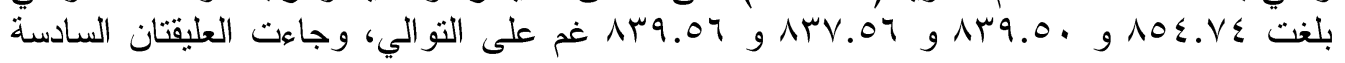

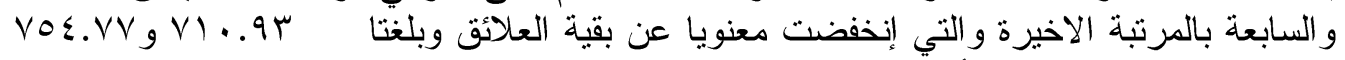

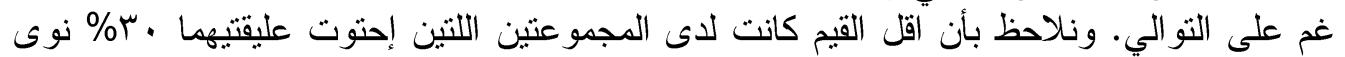
التمر مع أو بدون إضافة خميرة الخبز وقد يعزى السبب إلى ان حملان العليقتين إستهلكتا اقل كمية من العلف الف الت حسابيا و اقل كفاءة تحويل غذائي حسابيا ايضا بسبب ارتفاع نسبة نوى التمر وبالتالي انخفضت

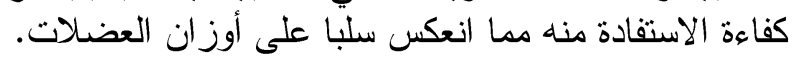

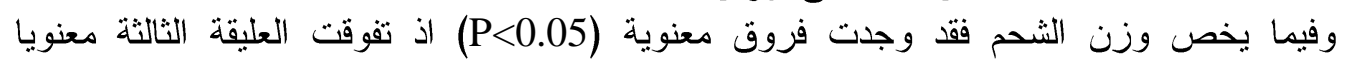
(P<0.05)

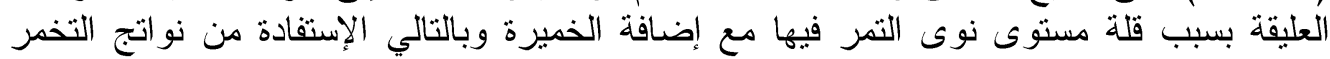

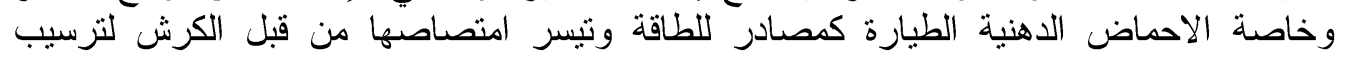

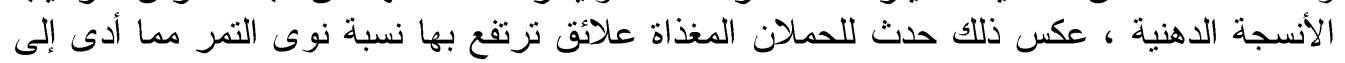

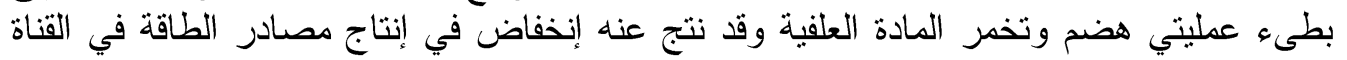
الهضمية للحملان.

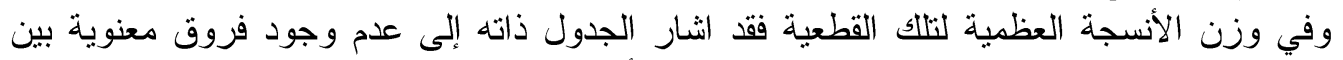

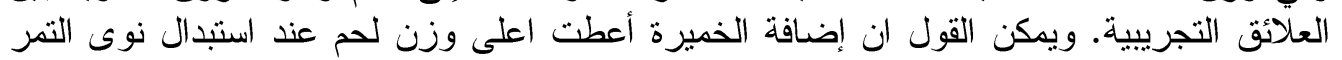

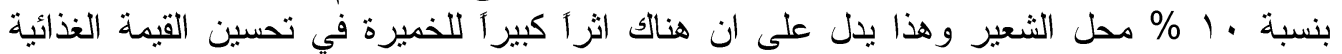

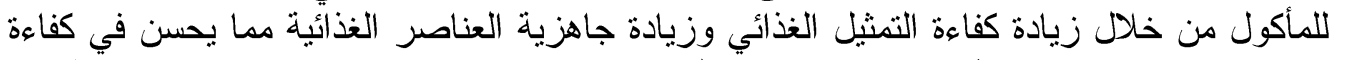

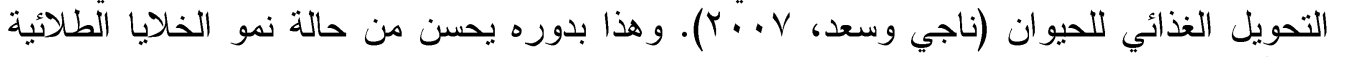

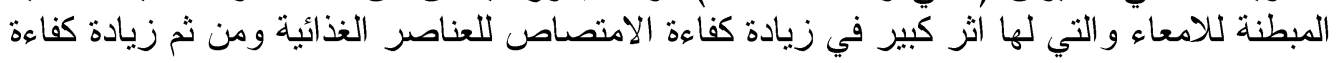

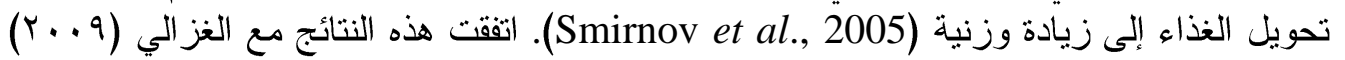

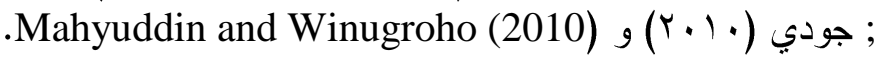

Fayoum J. Agric. Res. \& Dev., Vol. 33, No.2, July, 2019 
جدول (†). متوسط وزن الأسجة العضلية و الثحمية والعظمية لقطعية الاضلاع للعلاثق

\begin{tabular}{|c|c|c|c|}
\hline \multicolumn{3}{|c|}{ الصفات \pm الخطأ القياسي } & \multirow[t]{2}{*}{ العلاثق } \\
\hline وزن العظم (غم) & وزن الشحم (غم) & وزن اللحم (غم) & \\
\hline$\sum 9.97 \pm r r \leqslant . T_{0}$ & br^... \pm ro1.. & b $\leqslant r . r) \pm 10 \leqslant . V \leqslant$ & الاولى (السيطرة) \\
\hline \multirow{2}{*}{$09.9 V \pm r V \varepsilon \ldots$} & \multirow[t]{2}{*}{ brv.s. \pm roT.0. } & \multirow{2}{*}{ bミ1.rা $\pm \wedge r q .0}$. & الثانية \\
\hline & & & إحلال · 1\% نوى التمر محل الثعير بدون \\
\hline \multirow[t]{2}{*}{ rr.00 \pm rqV.Vo } & \multirow{2}{*}{ arr.01 \pm Y^..00 } & \multirow{2}{*}{ a $\leqslant \vee .09 \pm 9 r 1 . V}$. & الثالثة \\
\hline & & & إحلا · 1\% نوى التمر محل الثعير مع \\
\hline \multirow[t]{2}{*}{$\varepsilon 0.9 \cdot \pm\{.1 \ldots$} & \multirow[t]{2}{*}{ bYo.l. \pm YYI.TV } & \multirow[t]{2}{*}{ 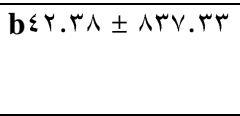 } & الر الرةة \\
\hline & & & إحلال · r\% نوى التمر محل الثعير بدون \\
\hline \multirow[t]{2}{*}{ NV.l. \pm rV৭.Vo } & \multirow[t]{2}{*}{ brY.Or \pm YYr.T9 } & \multirow[t]{2}{*}{ b乞 $1.74 \pm 149.04$} & الخامسة \\
\hline & & & إحلال · r\% نوى التمر محل الثعير مع \\
\hline \multirow[t]{2}{*}{ MY.rY \pm MYO.YO } & \multirow[t]{2}{*}{ crl. $\Sigma V \pm r . r . \wedge r$} & \multirow[t]{2}{*}{$c \leqslant \varepsilon . r \leq \pm V \cdot . .9 r$} & السادسة \\
\hline & & & إحلال ، \%\% نوى التمر محل الثعير بلون \\
\hline rq.1. \pm rqs... & crq.1 $\leq \pm r 11 . r r$ & $c \leqslant Y . q 1 \pm V 0 \leqslant . V V$ & إحلال · r\% نوى التمر محل الثعير مع \\
\hline N.S & * & * & مستوى المعنوية \\
\hline
\end{tabular}

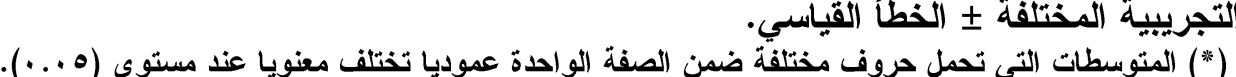
(N.S)

وزن الاهون المفصولة

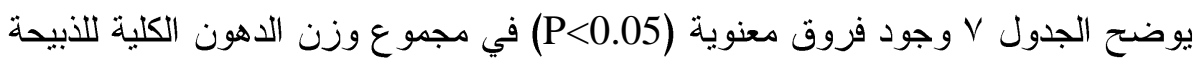

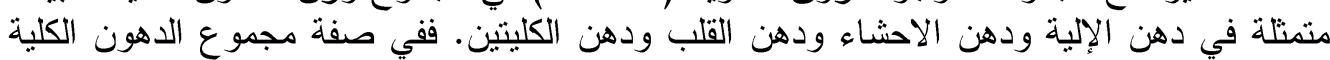

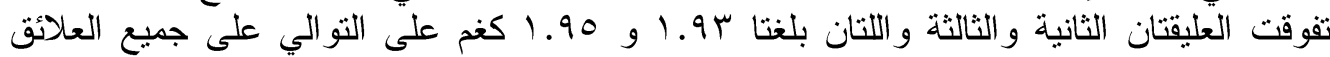

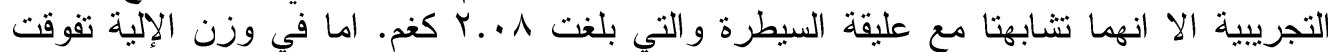

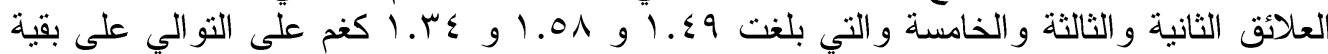

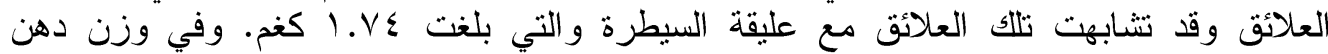

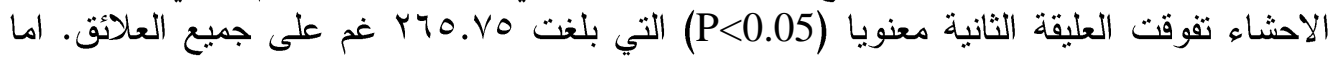

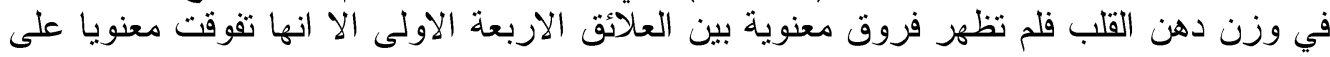

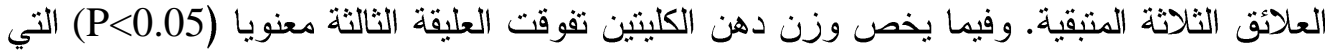

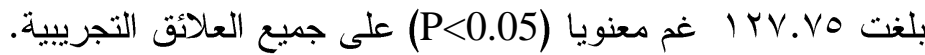

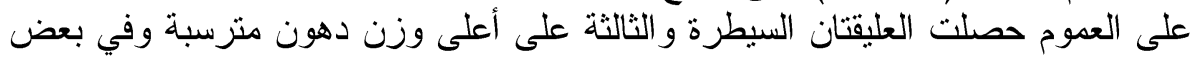

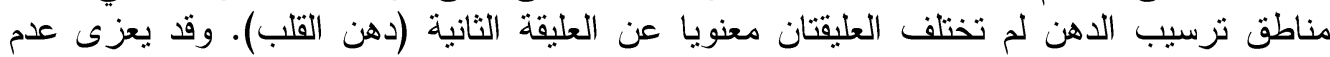

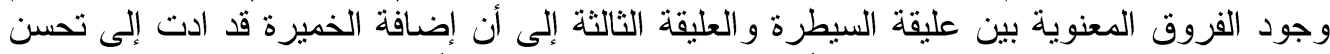

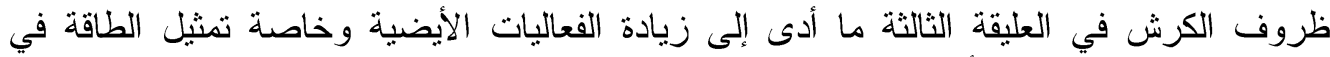

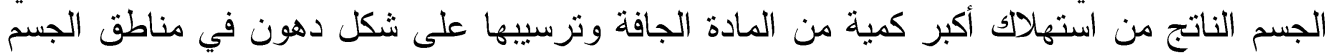

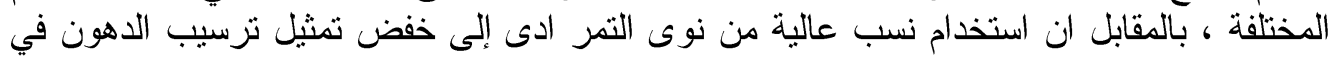

Fayoum J. Agric. Res. \& Dev., Vol. 33, No.2, July, 2019 
rq

مناطق الجسم المختلفة. وجاءت هذه النتائج متفقة مع (2008) .Titi et al ; الغز الي (9 ( . ( )

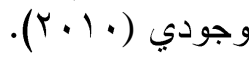

جدول (V). متوسط وزن الاهون المفصولة للعلانق التجريبية المختلفة 土 الخطأ القياسي.

\begin{tabular}{|c|c|c|c|c|c|}
\hline \multicolumn{5}{|c|}{ الصفات 土 الخطأ القياسي } & \multirow[b]{2}{*}{ 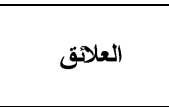 } \\
\hline وزن دهن الكليتين (غم) & وزن دهن القلب (غم) & وزن دهن الاحشاء (غم) & وزن دهن الإلية (كغم) & مجلموعة الاهونة & \\
\hline c Y.O \pm VY.O. & 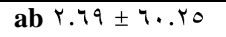 & bo.rr $\pm 199 . \mathrm{ro}^{\prime}$ & a $. \leqslant 0 \pm 1 . v \leqslant$ & a $\cdots \leqslant r \pm r . \cdot \Lambda$ & الاولى (السيطرة) \\
\hline b $0.79 \pm 99.10$ & $a \leq . \leqslant r \pm V_{0} . r_{0}$ & a $1.10 \pm$ r 10.10 & a..$\leqslant r \pm 1 . \leqslant 9$ & a $\cdot .4 r \pm 1.9 r$ & 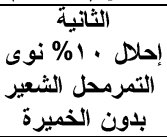 \\
\hline a $r .01 \pm 1 r v . v_{0}$ & ab $\leq .1 \wedge \pm\{\wedge .$. & b $\wedge . \Upsilon^{\prime} \pm 19 \varepsilon . . r$ & $a \cdot . r v \pm 1.01$ & a $\cdots \leqslant r \pm 1.90$ & 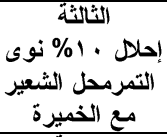 \\
\hline c $\leq .7 r \pm 7 V . T V$ & a $r . \leqslant v \pm 71.4 V$ & b $1.01 \pm 111 . r \mu$ & b $. .1 r \pm 1 . r V$ & b..1 $\leq \pm 1.01$ & 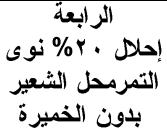 \\
\hline$c \leq . \Lambda \leq \pm V \leqslant .0$. & b Y. $\{\leqslant \pm \leqslant$ Y.YO & c $0 . \varepsilon \leqslant \pm 1$ rV.ro & a..ro $\pm 1 . r \varepsilon$ & b $\ldots r v \pm 1.7$ & 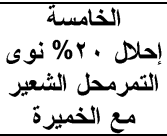 \\
\hline c r.09 $\pm 04 . .$. & b r.rl $\pm \leqslant r . v_{0}$ & $c A . r Y \pm 1 \leqslant \leqslant . r_{0}$ & b. . $\leqslant 0 \pm 1 . r \mu$ & b $\cdot r \lambda \pm 1 . \leqslant v$ & 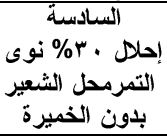 \\
\hline c $0 . \leqslant r \pm 04.0$. & b Y.AV $\pm \leq \varepsilon .0$. & c $А .74 \pm 1 \% r \ldots$ & b. . $\left\{\lambda \pm 1 . r_{0}\right.$ & b $\cdot . \leqslant 4 \pm 1 . \leqslant 1$ & 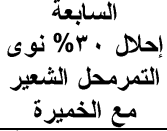 \\
\hline * & $*$ & * & * & * & مستوى المعنوية \\
\hline
\end{tabular}

(") المتوسطات التي تحمل حروف مختلفة ضمن الصفة الواحدة عموديا تختلف معنويا عند مستوى (0...).

(المصادر

الحريص، ناظم محمد جواد علي. (ع ا • Y). تأثير استخدام المعزز الحيوي (Probiotic) وخمبرة الخبز(Saccharomyces cerevisiae) مع الماء المعالج مغناطيسياً في بعض الصفات الإنتاجية

و الفسلجية لحمالن الأغنام العر ابي الذكرية. رسالة ماجستير - كلية الزر اعة- جامعة البصرة. الغز الي ، بشار نوري كاظم. (2009). تأثثر استخدام بعض الأعلاف الخشنة والمعاملة بالخميرة Saccharomyces cerevisiae الإنتاجية للحمان العواسية. رسالة ماجستير - الكلية التقنية- المسيب- هيئة التعليم التقني- العراق.

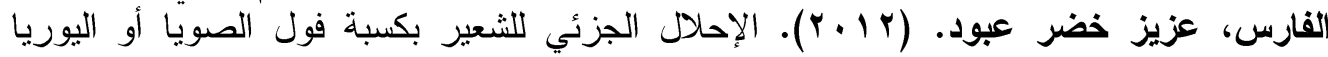
و إضافة خمبرة الخبز Saccharomyces cerevisiae إلى عليقة الحملان الذكرية العرابية واثرها في الاداء ونمو الاحياء المجهرية في الكرش. أطروحة دكتور اه ـ كلية الزر اعة ـ ـ جامعة البصرة. جودي، رشا علي. (2010). تاثير إضافة المعزز الحيوي إلى علائق مختلفة من نوى التمر في اداء الحملان العو اسية. رسالة ماجستير - الكلية التقنية- المسيب.

Fayoum J. Agric. Res. \& Dev., Vol. 33, No.2, July, 2019 


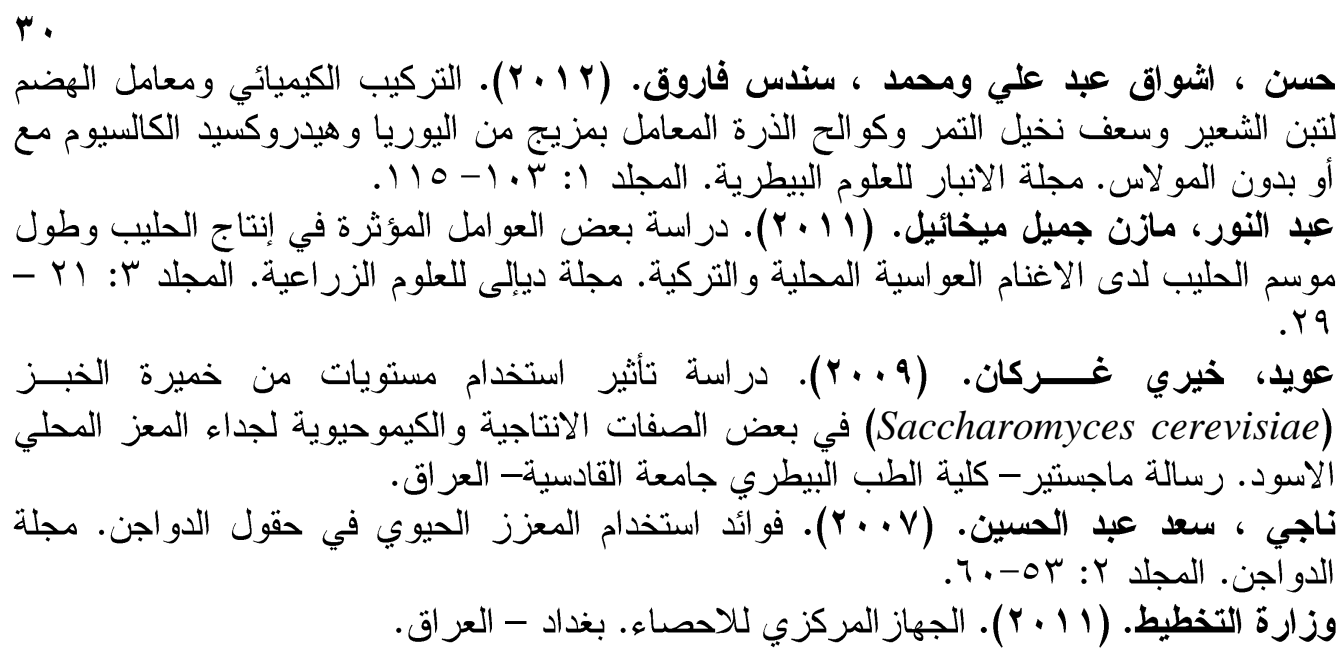

Abdul-Aziz, G. M., El Shaer, R. M., Fahmy, A. A., Shalaby, A. S., \& Abd El Gawad, A. M. (2001). Carcass quality of fattened sheep fed halophytic silage with non-conventional energy supplements in Egypt. Production Systems and Product Quality in Sheep and Goats. Eds Rubino, R. \& Morand-Fehr, P., Options Mediterraneennes: Serie A. Seminaires Mediterraneens, 46, 35-39.

Al-mahddawi, M. K. (2011). Effect of using different levels of protein in the diet and fattening period on body diemensions of Iraqi lambs. $J$. Diyala Agri. Sci., 3: 38-50.

Al-Sawaf, D. I. M. (2011). Qualitative and Quantitive Analysis of Amino Acids Isolated from Protein of Phoenix dactylifera and Determination of Molecular Weight by Gel Filtration (part I). Rafidain J. Sci., 22: 111-128.

Al-Shanti, H. A., Kholif, A. M., Al-Shakhrit, K. J., Al-Banna, M. F. and Showayb, I. A. (2013). Use of crushed date seeds in feeding growing Assaf lambs. Egyptian J. Sheep and Goat Sci., 8: 65-73.

Angulo, A. A., Valdes, Y. S., Carrillo-Muro, O., Castro-Perez, B. I., Barreras, A., Lopez-Soto, M. A. and Zinn, R. A. (2013). Effects of feeding different levels of chromium-enriched live yeast in hairy lambs fed a corn-based diet: effects on growth performance, dietary energetics, carcass traits and visceral organ mass. Animal Production Sci., 53: 308-315.

Awadalla , I. M., Maareck, Y. A., Mohamed M. I. and Farghaly, M. S. (2002). Responses to partial replacement of yellow corn in rahmani lambs rations with ground date seeds on growth rate, digestion coefficients, rumen fermentation and carcass traits. Egyptian $J$. Nutrition and Feeds, 5: 139-154.

Fayoum J. Agric. Res. \& Dev., Vol. 33, No.2, July, 2019 
r)

Duggan, C., Gannon, J. and Walker, W. A. (2002). Protective nutrients and functional foods for the gastrointestinal tract. Am. J. Clin. Nutr., 75: 789- 808.

MAFF. (1975). Ministry of Agric. Fisheries and food dept. of Agric. and fisheries for Scotland energy allowances and feed systems for ruminants. Technical Bulletin, 33. First published.

Mahyuddin, P. and Winugroho, M. (2010). Effect of combination of yeast (Saccharomyces cerevisae + Candida utilis) and herbs supplementation in finishing diet on carcass characteristics of beef cattle. J. the Indonesian Tropical Animal Agriculture, 35: 251-256.

Oguz, F. K., Bugdayci, K. E. and Oguz, M. N. (2015). The Effects of Yeast Culture Products on Fattening Performance, Rumen Papilla Morphology, Some Blood and Rumen Fluid Parameters in Saanen Male Kids. Kafkas Universitesi Veteriner Fakultesi Dergisi, 21: 455461.

Payandeh, S. and Khafilzadeh, F. (2007). The effect of yeast (Saccharomycescerevisiae) on nutrient intake, digestibility and finishing performance of lambs fed diet based on dried sugar beetpulp. Pakistan J. Biol. Sci., 10: 4426-4431.

Saarela, M., Mogensen, G., Fonden, R., Matto, J. and Mattila-Sandholm, T. (2000). Probiotic bacteria safety, functional and technological properties. J. Biotechnol., 84: $197-215$.

Selmi, H., Khaldi, Z., Tibaui, G., Ben Gara, A., Rekik, B. and Rouissi, H. (2011). Nutritional preliminary characterization of some varieties of dates and palm downgraded as ruminant feed. Online J. Anim. Feed Res., 1: 73-76.

Smirnov, A., Perez, R., Amit-Romach, E., Sklan, D. and Uni, Z. (2005). Mucin dynamics and microbial populations in chickens small intestine are changed by dietary prebiotic and antibiotic groqth promoter supplementation. J. Nutr., 135: 187 - 192.

SPSS. (1998). Statistical Packages for Social Sciences. Version 9.01.Manual. U.S.A.

Titi, H. H., Abdullah, A. Y., Lubbadeh, W. F. and Obeidat, B. S. (2008). Growth and carcass characteristics of male dairy calves on a yeast culture-supplemented diet. South African J. Animal Sci., 38: 174-183.

Fayoum J. Agric. Res. \& Dev., Vol. 33, No.2, July, 2019 


\title{
SUBSTITUTED DIFFERENT RATIOS OF CRUSHED DATE PITS FOR THE BARLEY WITH OR WITHOUT ADDITION OF SACCHAROMYCES CEREVISIAE ON SOME CHARACTERISTICS OF THE CARCASSES OF ARABI LAMBS
}

\author{
K. H. Muhana*; A. F. AL-Jassim**; M. A. Tahir ** \\ *Department of Animal Production, Shatrah technical Institute, Southern \\ Technical University. \\ Kareemhammadi17@gmail.com \\ **Department of Animal Production, College of Agriculture, University of \\ Basrah,
}

\section{Summary}

This study was conducted in the Animal Farm of the College of Agriculture - University of Basrah. A total of 28 Arabi male lambs were used, with average age of 5 months and mean body weight of $21 . \leqslant \vee \pm 1.33 \mathrm{Kg}$. Lambs were distributed equally and randomly into 7 nutrition rations to conduct growth and fattening of Arabi lambs which lasted for 90 days. The experimental rations were as follows:

Ration-1- (control) - fed on concentrate ration only by $3 \%$ of body weight. Ration-2- fed on $90 \%$ of the control ration and $10 \%$ of ground date stones substituted for the barley.Ration-3- lambs fed on the ration 2 with addition of 3 grams of bakery yeast (Saccharomyces cerevisiae) / Kg of feed. Ration-4fed on $80 \%$ of the control ration with substitution of $20 \%$ of date stones for barley. Ration-5- lambs fed on the ration 4 with addition of 3 grams of bakery yeast per Kg of feed. Ration-6- fed on $70 \%$ of the control ration and $30 \%$ of date stones. Ration-7- lambs fed on the ration 6 with addition of 3 grams of bakery yeast per Kg of feed.

\section{Results were as follows:}

1- The highest weights of rib cut (rib 7-12), leg cut and loin cut were for animals in ration $3(1.61,5.00$ and $2.40 \mathrm{Kg}$ respectively) than other rations but in the loin cut, the ration 3 was similar to the control ration $2.16 \mathrm{Kg}$.

2 - For body measurements, the highest leg circumference were recorded for animals in rations 2 and $3(35.00$ and $36.50 \mathrm{~cm}$ respectively) than other rations but these rations were similar to the control ration. While the highest rib eye muscle area was for carcasses of animals in ration $3\left(14.41 \mathrm{~cm}^{2}\right)$ but this value did not differ significantly from that of the control animals $\left(13.45 \mathrm{~cm}^{2}\right)$.

3 - In the case of some carcass muscle weights, the muscle Supraspinatus weight were higher in the animals of the ration $3(176.67 \mathrm{~g})$ but it was similar

Fayoum J. Agric. Res. \& Dev., Vol. 33, No.2, July, 2019 
to control ration (164.25 g). Weight of the muscle Biceps femoris was higher in the carcasses of animals in ration $3(625.75 \mathrm{~g})$.

4- Carcasses of animals in ration 3 recorded the highest dissected lean meat $931.70 \mathrm{~g}$ and fat $280.55 \mathrm{~g}$ in rib cut, but no significant differences were registered for dissected bone weight among the different rations.

5- Mean weights of separated fat of carcasses were higher for animals of the 2 and 3 rations (1.93 and $1.95 \mathrm{Kg}$ respectively) in comparison with animals of other rations but these rations were similar to the control ration $2.08 \mathrm{Kg}$.

Keywords: Bakery Yeast, Date Pits, carcasses characteristics, Arabi Lambs.

* The research is based on the $\mathrm{Ph}$. D. Thesis, of the first researcher.

Fayoum J. Agric. Res. \& Dev., Vol. 33, No.2, July, 2019 